\title{
Les jeunes et le lien social. A propos de la violence scolaire
}

\section{Marc Barthélémy}

\section{(2) OpenEdition}

1 Journals

\section{Édition électronique}

URL : http://journals.openedition.org/corpsetculture/482

DOI : 10.4000/corpsetculture.482

ISSN : $1777-5337$

\section{Éditeur}

Association Corps et Culture

\section{Édition imprimée}

Date de publication : 1 juin 1998

ISSN : 1268-5631

\section{Référence électronique}

Marc Barthélémy, «Les jeunes et le lien social. A propos de la violence scolaire », Corps et culture [En ligne], Numéro 3| 1998, mis en ligne le 24 septembre 2007, consulté le 10 décembre 2020. URL :

http://journals.openedition.org/corpsetculture/482 ; DOI : https://doi.org/10.4000/corpsetculture.482

Ce document a été généré automatiquement le 10 décembre 2020.

(c) tous droits réservés 


\title{
Les jeunes et le lien social. A propos de la violence scolaire
}

\author{
Marc Barthélémy
}

Introduction

1 Aborder la question du lien social et de la jeunesse suppose au préalable que soit construite la catégorie « jeunesse ». En effet, chaque théorie du lien social renvoie à une conception de la socialisation et donc à une définition de ce qu'est un jeune par rapport à un adulte. La socialisation considérée comme intériorisation du social, fera pencher l'analyse plutôt vers une crise de la transmission de normes. Considérée comme produit des interactions, l'attention se portera sur la crise des compétences à s'adapter et à construire des situations qui constituent l'expérience sociale.

2 De plus, les catégories habituellement mobilisées ne sont pas toujours opératoires pour séparer le jeune de l'adulte, le normal du déviant, l'inclus de l'exclus. Une période d'incertitude semble s'être étendue à l'ensemble de la jeunesse et plus seulement à ceux que le cumul des handicaps désigne comme jeunes en difficulté. Les politiques d'insertion voient leurs « dispositifs » débordés, poussés à redéfinir leurs missions. Le risque de déboucher sur une analyse en terme de manque, ou de vide social, et la certitude de l'insuffisance d'une problématique des stades ou des âges de socialisation, conduisent dans un premier temps à une analyse des représentations actuelles de la jeunesse, ou plutôt des jeunesses.

3 L'état «normal » de la jeunesse aujourd'hui est d'être scolarisé. Parce qu'elle est un service public et à ce titre s'adresse à tous, l'école peut donner une chance $\mathrm{d}$ 'intégration à celui qui respecte la règle du jeu. Un consensus autour d'un modèle de fonctionnement, a établi des normes auxquelles chacun doit se conformer. Celui qui ne s'y soumet pas ne comprend pas son intérêt. Ce déviant doit être «re-mobilisé » sur le front du savoir car il compromet ses chances d'intégration, ou celle de ses camarades. C'est pourquoi, outre le fait qu'elle a changé de nature et n'a pas de sens apparent, la violence qui apparaît dans les établissements scolaires est devenue insupportable. Souvent ressentie comme injuste envers les plus défavorisés elle n'a aucune légitimité critique. Pourtant l'école propose des épreuves de nature différente selon les milieux 
d'origine. Après l'école élémentaire le parcours scolaire n'est qu'une suite de "désajustements successifs » (Dubet F., Martucelli D., 1996) pour ceux dont la famille ne mobilise pas suffisamment de ressources pour assurer la continuité de la socialisation scolaire. La violence à l'école est donc toujours aussi une violence de l'école qui distribue ses certificats à exercer une autorité légitime (Bourdieu P., 1979).

Pour un phénomène qui n'est pas si récent, pourquoi donc un tel sentiment de crise? Les enquêtes montrent un développement exponentiel des incidents et une forte corrélation entre violence scolaire et «exclusion perçue " (Debarbieux E., 1996) qui renvoie à « la fracture sociale ». Les recherches tentent de mettre à jour les variables qui permettent d'expliquer l'intensité des manifestations. Mais plus largement la violence à l'école est le signe de l'installation durable d'un nouveau rapport de force, l'analyseur institutionnel de la vulnérabilité du lien scolaire défini par J. Pain (1992) comme une réalité psychosociologique constituée des représentations des familles, des jeunes et de l'institution. Les actes de violence scolaire s'inscrivent dans un contexte, Produits dans un cadre institutionnel et local, ils ont un sens pour leurs auteurs et ceux qui les subissent.

5 L'objectif de cette contribution vise à re-situer ces actes, et à participer à l'étude des civilités et de la citoyenneté scolaire ordinaire, en articulant les approches de champs de recherche souvent disjoints. Il est nécessaire de revisiter les lieux afin d'apprécier leur capacité à tisser du lien, et d'observer les dynamiques qui alimentent ce qui pourrait être désigné comme la désaffiliation du scolaire.

6 Après une définition de la situation de la jeunesse, la violence scolaire sera considérée comme une manifestation banale de la crise du lien. Seront esquissées alors quelques contradictions à l'enseignement des compétences citoyennes mobilisées à son secours, La jeunesse : une affaire de représentation qui néglige la diversité de ses figures

7 Il est devenu banal d'envisager la question sociale sous l'aspect d'une rupture entre les conduites des jeunes et les attentes des institutions publiques chargées de les encadrer. La société se penche avec angoisse sur «la jeunesse», qui est presque devenue une catégorie à intégrer, mais qui échappe aux dispositifs mis en place. Préoccupation qui témoigne d'une évolution des représentations de la place des jeunes et du statut de la jeunesse : «ils sont les enfants de notre société alors que nous étions les enfants de nos parents $»^{1}$. Mais il semble y avoir autant de jeunesses que de points de vue sur elle.

8 La jeunesse c'est "un mode de vie». Elle est douée d'une socialité hédoniste et généreuse, animée par une pulsion dionysiaque (Maffesoli M.), et le goût de la dépense. Elle est présentée comme un monde à part, doté d'une culture adolescente (Fize M., 1994). Mais même si les jeunes ont leurs propres réseaux, ils sont en tête de presque toutes les pratiques culturelles: sport, danse, cinéma, musique, fréquentation des musées, expositions, théâtre, ou encore pratique de l'écriture ou de la lecture ( $84 \%$ de lecteurs pour les 15-24 ans contre 75\% pour le reste de la population) ${ }^{2}$.

9 La jeunesse c'est « un processus de maturation ». Les rôles et le statut d'adulte sont moins qu'hier l'héritage d'une identité familiale, mais leur élaboration par expérimentation demande du temps. Celui des classements (Mauger G., 1995) scolaires, professionnels, relationnels, culturels, amoureux ${ }^{3}$. Le temps conflictuel de la négociation identitaire (Dubard C., 1991: 115) au point de rencontre entre ce que le sujet veut être et ce que le monde lui permet d'être ${ }^{4}$ (Bourdieu : 1979, p. 123). Le temps des pratiques « ordaliques ", à défaut de rites ${ }^{5}$ de passage (Le Breton D., 1991), des défis 
qui permettent d'acquérir un statut dans le groupe, et de vérifier si l'on a la chance avec soi.

La jeunesse c'est « un nouvel âge de la vie » (Galland O., 1991). Il recouvre une échelle de situations intermédiaires qui correspondent à la flexibilité des formes d'entrée dans la vie adulte, dans le monde du travail, les systèmes de protection sociale, les structures de formation etc. Les transitions de l'école à l'emploi, et de la famille d'origine à la création d'une nouvelle famille se sont considérablement allongées.

La jeunesse c'est "une catégorie à intégrer ». Elle est particulièrement touchée par le chômage, génération sacrifiée, menacée d'exclusion. Le terme est un peu galvaudé, c'est « un mot valise » qui recouvre des situations diverses. Cependant, l'exclusion n'est plus la face cachée de la modernité (le fou, le prisonnier...). De périphérique, elle est devenue centrale et fait de chacun un exclu potentiel. Ainsi la forte mobilisation contre le C.I.P. a pu être expliquée par un sentiment de communauté d'intérêt entre ceux qui craignent l'avenir précaire et ceux qui « en galère » l'éprouvent déjà. Les jeunes payent l'incapacité politique à penser le travail comme pourvoyeur de dignité. Ils sont les plus touchés par le temps partiel subi et leur insertion par l'emploi "flexible » est en augmentation. Mais, la fortune de l'idée de fracture, de chute, ou l'évocation d'un équilibre instable entre un pôle «in » et un pôle « out " (Touraine) ou entre centre et périphérie (Dubet - Lapeyronnie) faussent la représentation d'un processus progressif qui de la vulnérabilité conduit à la « désaffiliation » (Castel R., 1991).

12 Les jeunes participent à des réseaux hétérogènes. Ils connaissent des formes d'inscriptions multiples dans les rapports sociaux qui leur permettent parfois de retraverser la zone d'incertitude scolaire. Inversement, parce qu'il est un vecteur de sensibilité « sociétal » (Pain J., 1992), à travers le lien scolaire, l'expérience de sa future place sociale peut être faite.

13 Les jeunes vivent ainsi de plus en plus tôt le bouleversement des rapports public-privé et la lutte des places (De Gaulejac, Taboada Léonetti I., 1994). Faire de "soi-même un but à atteindre " exige la constance d'un individu responsable dont «le moi est le principal fardeau » (Sennet R., 1979). Des « individus incertains » (Ehrenberg A., 1995), convaincus qu'ils ne doivent leur épanouissement qu'à eux-mêmes sont sommés de se mettre en projets, de «montrer leur intérieur psychique» (Chambat P., 1993). Le paradigme du projet individuel (dès le collège) bouscule ainsi le processus «d'individuation» qui, selon simmel, doit correspondre à une sociabilité choisie. Quand le sens des études ne va pas de soi, que la culture adolescente s'autonomise, les élèves (les collégiens notamment) se réfugient dans une gestion conflictuelle de leur " face », alternant conformisme et authenticité.

La jeunesse ce «n'est qu'un mot ». Bourdieu (1984) dans un article célèbre, dénonçait à la fois les professionnels qui construisent une catégorie pour la prendre comme objet de recherche ou comme cible commerciale, et l'abus de langage qui occulte les antagonismes de classe qui la traversent. Autrefois la tolérance populaire aux excès de sa jeunesse, prenait sa source dans l'idée qu'ils iraient bien assez tôt prendre leur place à l'usine. Mais aujourd'hui les difficultés de ceux qui cumulent les « rapports sociaux d'exclusion » par la convergence à des degrés divers de l'échec scolaire, du chômage, de l'absence de droits civiques, et qui subissent leur « relégation dans certains quartiers de banlieue» (Paugam S., 1991), questionnent la capacité d'intégration du modèle républicain. La citoyenneté, le manque de civisme sont interrogés. Pour cette « autre 
jeunesse » (Dubar C., 1987) la crise de socialisation ne peut plus être présentée comme une désadaptation fonctionnelle temporaire.

Dans les Z.U.P., la question « sociale » est rabattue sur la question « urbaine ». Pourtant, bien peu des maux dont souffrent ces quartiers, relèvent du cadre où l'on s'évertue à les circonscrire. Les "adultes" parlent de rappel de la loi, des règles sociales. Les jeunes parlent de racisme, d'incompréhension et d'humiliation. Entre « rêve de fuite et défense d'un territoire assiégé » (Bachman C., $1994: 144)$ leur " frustration » (Galland 0., 1991) se renforce de la « déliaison » entre promesse d'intégration et réalité sociale et scolaire. Et la difficulté à reconnaître l'adversaire social tourne leur " rage » contre eux-mêmes ou leur propre quartier (Dubet F., Lapeyronnie D., 1992).

Tout discours sur «la jeunesse » rassemble donc, sous un même concept, des univers sociaux très polarisés. Mais, reconnaître «des jeunesses" ce n'est pas prétendre qu'elles n'ont rien en commun. En témoigne l'allongement de la période et la grande flexibilité des seuils d'entrée dans l'âge adulte, la socialisation des jeunes " entre eux » qui concurrence les transmissions verticales traditionnelles, ou encore des modes de sociabilité peu reconnus dans des "espaces intermédiaires ${ }^{6}$ (Nicole-Drancourt C., Roulleau-Berger L., 1995). La recomposition des figures de la jeunesse suit cependant la fragmentation du lien social.

17 Des liens existent et d'autres sont créés. La production des signes et des objets emblématiques permet aux « tribus urbaines » de se repérer (Maffesoli M., 1988). Mais, sans négliger la mutation des principes (éthiques) qui fondent l'être ensemble, l'importance est moins accordée ici à la manière de mobiliser un capital social, qu'aux tensions que provoque l'allongement de la période d'incertitude. Avec la scolarisation massive et la dissociation du titre scolaire de la structure de l'emploi, une période plus ou moins longue d'ajustement s'ouvre après la scolarité. Ce constat influence le regard porté sur la période scolaire. N'est-elle pas préparatoire à l'enchevêtrement d'autres temps sociaux comme le chômage, les petits boulots, la formation ou la poursuite d'études?

Le lien scolaireRéférence paradoxale : on attend de l'école à la fois tout et rien

18 Cette épreuve bien connue des familles, qui faisait relativement l'accord entre générations, recouvre une réalité nouvelle, angoissante. Le lien scolaire est menacé d'éclater sous la pression des représentations divergentes des acteurs de l'intérieur de l'école, mais aussi de l'extérieur. Historiquement la contribution de l'école au lien social passait par une rupture qui devait libérer l'individu de ses attaches domestiques. L'accord se faisait sur des principes légitimes, une construction d'une "paix des objets » (Dérouet J.-L., 1992) qui organisait la neutralité de l'espace public... Cet accord est dénoncé. L'école est comparée à un supermarché (Ballion R., 1993) dans lequel les familles, en concurrence, mettent en œuvre des stratégies d'acquisition de qualification.

19 Cette demande de plus en plus forte de diplômes se conjugue à une perte du sens de l'école en terme de culture et de compréhension du monde. Du côté des élèves le malentendu apparaît dans une approche utilitariste des matières ( «la musique ça sert si on veut devenir musicien »). Mais, lorsque l'état normal de la jeunesse est d'être scolarisé, comment se résoudre à l'échec. Le maintien dans le système scolaire ne fait pas longtemps fonction de lien aux «exclus de l'intérieur» (Bourdieu P., 1993). Ne voulant pas renoncer à des diplômes qui sont en relation avec la qualité des emplois et leur rémunération, ils se réfugient (au mieux) dans le respect des consignes et un 
rapport utilitaire à l'école (Charlot B., 1997). Les enseignants se demandent alors comment les aider à construire le sens de l'expérience scolaire (Rochex J.-Y., 1995).

A côté des débats autour du niveau et de l'échec, les pouvoirs publics embarrassés par la « crise de la jeunesse » et la montée de la violence redécouvrent l'apprentissage du civisme, de la citoyenneté. Beaucoup comme Meirieu (1995) proposent de surmonter le risque d'un clivage en interrogeant conjointement la didactique des apprentissages et de la socialisation. Penser la globalité de l'acte scolaire afin d'envisager la transmission de savoir sous un angle qui permette le partage d'une commune humanité.

L'établissement scolaire : un dispositif central d'intervention

21 Parce que la jeunesse pose problème, dans les stades, les transports en commun, le débat sur la sécurité urbaine s'est déplacé vers l'école. Elle est chargée d'une mission de « requalification» des territoires en crise mais aussi de la mise en œuvre d'un civisme ordinaire. L'établissement scolaire est devenu un dispositif central d'intervention pour repenser, la ville, la citoyenneté, la violence, etc.

L'implication des acteurs

22 L'approche contemporaine du lien social s'appuie sur l'implication des acteurs. J.-L. Dérouet (1992) souligne que depuis Fontanet (pourcentage d'autonomie des établissements), en passant par Beulac (notion de projet), jusqu'à la loi d'orientation de 1989, un mouvement vers le local et les élèves se dessine. La loi de 89 promet l'intégration plus que la mobilité sociale. Les grands acteurs à l'échelle des établissements, participent à la politique locale. Les petits acteurs à l'échelle de la classe participent à la gestion des capacités et des droits pour une « juste» orientation. Des parcours différenciés sont proposés selon des aptitudes et des compétences qui dépendent cependant du rapport au savoir et du degré de mobilisation des familles (Thérail J.-R, 1995).

23 Les municipalités se mobilisent. L'hypothèse est que l'école, représentant un secteur public "requalifié ", peut entraîner une dynamique de coopération avec le quartier (aide aux devoirs etc.) et tisser autour d'elle un dispositif éducatif plus large. Ouverture nécessaire de l'école sur la cité sans pour autant qu'elle se "déscolarise » et perde la spécificité de sa fonction. Si la régulation devient plus rapide et plus souple à l'échelle locale, cette diversification de l'offre d'enseignement risque de donner lieu à des inégalités nouvelles suivant les ressources des villes et leur degré de mobilisation en faveur de l'enseignement (Henriot-Van Zanten A. 1994). Mais ces évolutions correspondent à un souci d'adaptation à l'hétérogénéité des publics. Les années 80 ont vu le multiculturalisme chercher sa place dans l'espace public. Résultat de la prise en compte d'un citoyen situé (Bonny Y. 1995) et non plus abstrait, ou dissolution de la citoyenneté vers une urbanité plus "fonctionnelle»? Le civisme républicain s'est ouvert à de nouvelles formes de participation démocratique. Les dispositifs d'action (Z.U.P, C.C.D.P, D.S.Q., Z.E.P...) fonctionnent à « l'implication » et « constituent à ce titre autant de communautés de responsabilité » (Donzelot J., 1991). Certains y voient une évolution démocratique du système républicain, un nouvel esprit public, d'autres le risque du compromis local, et la perte de transcendance du politique au profit des groupes de pression. Mais instituer une certaine visibilité des acteurs (condition de publicité de l'espace pour $\mathrm{H}$. Arendt) ne va pas sans poser problème. Entre ouverture et clôture l'école doit redéfinir l'identité de ses missions? ${ }^{7}$.

Le lien scolaire est fragilisé par les tensions urbaines 
Repérée à son tour comme une zone de turbulence, entre la demande sociale d'efficacité et l'aggravation de la situation, l'école ne peut plus taire ses difficultés. Elle n'est plus un sanctuaire protégé, et ses échanges avec la ville ou le quartier provoquent des processus de qualification ou de déqualification mutuels (Defrance B., 1988). On tente d'analyser « l'effet établissement » (Duru-Bellat M., Mingat A., 1988). En parallèle avec la lutte contre l'échec et l'apathie, on mobilise les acteurs pour ne pas amplifier les effets de facteurs externes à l'institution.

La violence scolaire est aujourd'hui mieux cernée. Elle se distingue de celle du quartier par un fort taux de résolution des conflits et une entrée plus fréquente en régime de justice. Peu de graves affaires restent impunies. Mais à côté de phénomènes relativement maitrisés, d'autres sont plus insaisissables. Ce sont, rapporte B. Charlot ${ }^{8}$ : « les incivilités » (J.-P. Payet), la « malmenance » (J. Pain), « les transgressions molles » (R. Ballion) et autres brutalités. Leur banalisation crée un climat de tension, l'angoisse d'actes plus graves. Ces phénomènes génèrent et amplifient la peur du collège chez les plus jeunes. Peu d'enquêtes se hasardent à chiffrer les délits, tant cette forme d'anomie est difficile à cerner, mais les enseignants témoignent d'un profond malaise.

Dans le registre des atteintes aux civilités, et pour ce qui concerne les rapports entre pairs, les jeunes se plaignent avant tout du manque de respect, puis des affaires abîmées, du chantage, des coups, du racisme, du racket, et enfin d'agressions et de harcèlement sexuel ${ }^{9}$. La mise en scène de ces actes conduit les études qui s'intéressent $\mathrm{au}$ " couple victimologique » agresseur-agressé, à proposer l'idée que ces attaques sont moins dirigées contre les personnes elles-mêmes que contre les règles qui assurent le maintien du lien social (Villerbu M., 1994). Il s'agit de semer le doute de toute légitimité, de déstabiliser le groupe ou des fonctions assurées en jouant avec la crainte. La création d'un climat d'insécurité et l'incertitude des cibles visées, développent par la passivité exigée des victimes réelles ou potentielles, une dévalorisation de soi et des normes morales. Le respect de la parole donnée, la possibilité d'agir, de s'engager sont mis en doute (Ricœur P, 1990 : 309-312). Cette situation conduit aussi les élèves à la perception d'un sentiment exagéré d'insécurité (Ballion R., 1993).

La clarification de ce que recouvre le terme de "violence scolaire » est un préalable pour apprécier son importance. Cependant la plupart des recherches proposent de dépasser une évaluation de celle-ci en fonction d'une position des actes sur une échelle de gravité. Leur point de vue heuristique consiste à situer les actes graves dans un ensemble plus large. Dans les civilités se jouent les droits et les devoirs concrets des individus, la possibilité d'un monde commun. Les travaux consacrés à la sécurité urbaine aux Etats-Unis montrent que leur non respect est le point de départ d'une spirale du déclin (Wilson J.-Q., Kelling G.-L., 1994). De la même manière la violence scolaire doit être rapportée à une "mécanique des incivilités » (Dumay J.-M., 1994), et être articulée dans une perspective interactionniste, au contexte institutionnel dans lequel elle prend sens, en pensant la frontière école-quartier comme problématique et variable (Payet J.-P., 1992).

Dès lors, il faut remarquer que ce phénomène prend une dimension politique au sens d'une interrogation de la citoyenneté des jeunes saisie dans l'évolution des civilités. Il n'est donc pas limité aux établissements dits difficiles, bien que le lien exclusionviolence perçue soit perceptible dans les analyses factorielles de Debarbieux (1996). Il renvoie à une mutation globale du rapport à la jeunesse et de l'expérience scolaire dont les règles de base sont remises en question. 
La violence une occasion de dire le droit principes du droit dans la définition et l'application des règles scolaires et dans la gestion des conflits. Le droit postule en effet «un ordre de représentation du lien social ». il articule avec l'aide des lois, des concepts liés au statut de la personne (identité, citoyenneté) avec des concepts liés à des valeurs (liberté, équité). 

préalable. Une règle peut renvoyer à une autre, le fonctionnement du droit sollicite un fondement extra-juridique, quelque chose du registre du "vouloir vivre ensemble ». "On ne peut fonder sa confiance sur le droit puisque celui-ci la présuppose» dit J. Roman (1994). Cela vaut au niveau macro-sociologique. Pour les théoriciens du contrat social le contrat entre individus souverains devait être articulé à une vision rationnelle de l'être ensemble, une «religion civile» (Roman J., 1994) («religare»: relier). Incapable d'aller au-delà d'un fondement juridique la pensée libérale est en échec: le contrat ne fait pas le lien. Cela vaut aussi au niveau micro-social. La théorie des jeux en fait la démonstration. Elle éclaire la rationalité des stratégies des acteurs et leurs capacités d'anticipation, mais elle montre aussi que ces sujets rationnels, par manque de confiance ne maximisent pas leurs gains. La poursuite du seul intérêt condamne chacun à ne pas jouer le jeu... si l'on ne peut être sûr de son partenaire.

Aussi, l'essence du lien tient-elle dans la volonté de s'impliquer dans un accord qui suppose une confiance mutuelle. Sur ce socle peut alors s'exercer l'idéal d'équité. Celui- 
ci dépend de la capacité à adapter les règles en vigueur lorsque l'on se rend compte qu'elles ne sont pas aussi justes qu'elles le paraissent ${ }^{11}$. Cette relation de confiance relève-t-elle du don pur ou d'une rationalité élargie? (agapè chrétienne ou philia grecque?).

La seconde hypothèse est la plus crédible, elle va dans le sens des travaux d'Alain Caillé (1994). L'attitude responsable d'un don (par exemple de droit, de citoyenneté pour la jeunesse) ne peut exister qu'avec l'oubli de ce qui l'a rendu capable de don (C'est-à-dire un premier don de droit, de citoyenneté). Mais la structure de réciprocité reste sousjacente, car l'intérêt du donneur est dans « la prise de risque » (Godbout J., Caillé A., 1992), et la possibilité de découvrir une réciprocité cachée, ou d'en créer une nouvelle. Vers une réciprocité généralisée (Temple D., Chabat M., 1995).

Conclusion

La violence est la conséquence de l'incapacité de la collectivité à s'auto-réguler et à définir les normes qui permettent l'accord. L'affaiblissement du lien scolaire qui en résulte amplifie le désarroi des acteurs d'une société dont les rôles et les statuts "flottent », surtout chez la jeunesse qui doit unifier une identité morcelée. Dans ce contexte le rappel du droit et de la loi sont insuffisants. La focalisation ici de l'attention sur les phénomènes de basse intensité de la violence scolaire n'est donc pas seulement un levier pour lutter contre la délinquance. Elle est aussi une manière d'observer à travers les logiques des acteurs comment l'accord sur ce qui est acceptable réussit encore à faire passer l'inacceptable.

$43 \quad 1$ - Il peut sembler banal de rappeler que le lien scolaire passe par les relations complexes qu'entretiennent la confiance et l'intérêt. Mais ces évidences doivent être actualisées à la lumière des nouvelles conditions d'articulation du scolaire et du social. Pour faire de l'école autre chose qu'un rouage intégrateur qui a montré ses limites, il faut la repenser dans les termes d'un espace public défini comme « un lieu de conflit, de dispersion et de chevauchement des appartenances" (Roulleau-Berger L., 1997). La citoyenneté scolaire, dont on déplore la centration autour de concepts fondamentalement juridiques et politiques, serait alors définie comme un mouvement de reconnaissance de l'autre, et de partage d'un territoire physique et symbolique qui soit le lieu d'un événement fondateur de mémoire. 2 - L'équité s'éprouve au travers de sphères de justice ${ }^{12}$ (Walzer M., 1997) et de situations soumises à un impératif de justification qui se réfèrent à des économies différentes de la grandeur ${ }^{13}$ (Boltanski L., Thévenot L., 1987). A l'école, les jeunes sont confrontés à différentes épreuves. Les plus évidentes sont les épreuves scolaires. Elles leur font vivre les contradictions d'une égalité entre eux, et la réalité simultanée d'un ordre, d'une hiérarchie dans cet état d'égalité. Mais d'autres Occasions de conflit existent. S'orienter dans la jungle du collège impose de passer par différents états: d'amour, de violence, de justice, de paix. Il faut mettre en oeuvre des modèles de " compétence au jugement ", appuyés sur des grandeurs légitimes, et se construire une identité en passant d'une situation de justice à une autre.

Le passage de la dispute pour défendre son intérêt à l'accord pour clore provisoirement cette dispute et entrer en « régime de paix » (Boltanski L., 1990 : 235) demande, en effet, des compromis équitables. L'ordre normatif d'une classe, d'une école ou d'une cité que l'on peut observer dans les activités jugées normales ou légitimes est ainsi le résultat d'une série d'accords et de désaccords, suivant la définition que les acteurs donnent à la situation et les ressources dont ils disposent. 

au sérieux la rationalité des acteurs, consistera à enquêter auprès de la jeunesse sur les conditions de justice qui lui sont faites, en considérant l'équité comme une condition de l'accord scolaire et non sa conséquence.

\section{BIBLIOGRAPHIE}

Audigier F, Lagelée G (1996) Éducation civique et initiation juridique dans les collèges, Paris, I.N.R.R

Bachman C. (1994) Jeunes et banlieue, Intégration et exclusion dans la société Française contemporaine, G. Ferreol (dir.) PU.L.

Ballion R. (1993) Le Lycée une cité à construire, Paris, Hachette-Éducation.

L'ordre scolaire menacé : le cas des lycées. Les cahiers de la sécurité intérieure, 92, mars 1994.

Boltanski L., Thévenot L. (1989) Les Economies de la grandeur, Cahier du CEE, série Protée, Paris, PUE.

Boltanski L. (1990) L'Amour et la justice comme compétence, Paris, Éditions Métailié.

Bonny Y. (1995) Les formes contemporaines de participation : citoyenneté située ou fin du politique ?, La citoyenneté aujourd'hui extension ou régression, P. Merle et E Vatin (dir), PUR.

Bourdieu P. (Il 979) La Distinction, Critique sociale du jugement, Paris, Éditions de minuit.

Bourdieu P (1984) Questions de sociologie, la jeunesse n'est qu'un mot, Paris, Éd. de Minuit.

Bourdieu P (1993) (sous la dir. de), La Misère du monde, Paris, Éditions du Seuil.

Caillé A. (1994) Présentation, Revue du Mauss $n^{\circ} 4$, Confiance interaction et théorie des jeux, La Découverte. Voir aussi Revue du Mauss $n^{\circ} 1, n^{\circ} 8$.

Castel R. (1991) De l'indigence à l'exclusion, la désaffiliation, Donzelot J. (dir.), Face à l'exclusion le modèle Français, Paris, Ed. Esprit. Du même auteur voir aussi : De l'exclusion comme état à la vulnérabilité comme processus, Affichard J. et De Foucauld J.-B. (dir.) (1992), Justice sociale et inégalités, Paris, Ed. Esprit.

Chambat P (Jan. 1993) La place du spectateur, Esprit.

Charlot B. (1997) Du Rapport au savoir. Elément pour une théorie, Anthropos/Economica.

De Gaulejac, Tabaoda Léonetti I. (1994) La Lutte des places, Paris, Desclée de Brouwer.

Debarbieux E. (1996) La Violence en milieu scolaire, Etat des lieux, (tome 1), E.S.F., Paris.

Defrance B. (1988) La Violence à l'école, Syros.

Defrance B. (Sept. 1995) École de la démocratie, démocratie dans l'école, Journal du droit des jeunes, 147

Defrance B. (Mars 1996) Colloque en Seine Saint Denis.

Dérouet J.-L. (1992) École et justice, Paris, Éditions Métailié.

Corps et culture, Numéro 3| 1998 
Donzelot J. (1991) Le social du troisième type, Face à l'exclusion le modèle Français, Esprit.

Dubard C. (1987) L'Autre jeunesse : jeunes stagiaires sans diplôme, P.U. de Lille.

Dubard C. (1991) La Socialisation, construction des identités sociales et professionnelles, A. Colin.

Dubet E., Lapeyronnie D. (1992) Les Quartiers d'exil, Paris, Seuil.

Dubet F., Martucelli D. (1996) A L'école, Paris, Seuil.

Durriay, J.-M. (1994) L'Ecole agressée réponse à la violence, Paris, Belfond.

Durut-Bellat M., Mingat A. (1988) Le déroulement de la scolarité au collège : le contexte fait des différences, Revue Française de Sociologie, XXIX.

Ehrenberg A. (1995) L'individu incertain, Paris, Calmann-Lévy.

Fize M (1994) Le Peuple adolescent, Paris, Julliard.

Galland 0. (1991) Sociologie de la jeunesse. L'entrée dans la vie, Paris, \&.Colin.

Godbout J., Caillé A. (1992), L'Esprit du don, La Découverte, Paris.

Henriot-Van Zanten A., L'action éducative des municipalités et la transformation du système éducatif, Migrant-Formation, 97, Op. cit, 7285.

La revue Migrant-Formation (1994) L'École dans la ville : Ouverture ou Clôture, 97, juin 1994

Le Breton D. (1991) Passions du risque, Métailié.

Maffesoli M. (1988) Le Temps des tribus, Paris, Méridiens Klincksieck.

Mauger G. (1995) Jeunesse l'âge des classements, Recherches et prévision, 40, Jeunesse le plus bel âge de la vie?

Meirieu P (1995) L'enseignant dans la crise, Spirales, 8: Spécial didactique, Lyon, CRIS UFRAPS.

Nicole-Drancourt C. et Roulleau-Berger L. (1995) L'insertion des jeunes en France, PUF.

Pain J. (1992) École violence ou pédagogie?, Matrice.

Paugam S. (1991) La Disqualification sociale, Essai sur la nouvelle pauvreté, Paris, P.U.F.

Payet J.-P (1992) Civilité et ethnicité dans les collèges de banlieue, Revue Française de Pédagogie, 101.

Pharo P. (1991) Politique et savoir-vivre, enquête sur le fondement du lien civil, Paris, L'Harmattan.

Ricoeur P (1990) Soi-même comme un autre, Paris, Seuil, Col. Points Essais.

Ricoeur P. (1995) Le Juste, Paris, Éditions Esprit.

Roché S. 0 996) La Société incivile, Paris, Seuil.

Rochex J. -Y. (1995) Le Sens de l'expérience scolaire : entre activité et subjectivité, Paris, PUF

Roman J. (hiver 1994-95) L'introuvable religion civile, Projet, 240, Paris.

Roulleau-Berger L. (Jv 1997) Procéduralisation, fragmentation et résistances juvéniles dans l'espace public, Citoyenneté identité, Doc INJEP Hors série nº 4 , Marly-le-Roi.

Sennet R., (1979) Les Tyrannies de l'intimité, Paris, Seuil.

Temple D., Chabat M. (1995) La Réciprocité et la naissance des valeurs humaines, Paris, L'Harmattan.

Thérail J.-P (1992) La Dynamique des Générations, L'Harmattan, 1995. Du même auteur, Réussite scolaire : la mobilisation des filles, Société contemporaine, 11 - 12. 
Villerbru M. (1994) (Dir), La Responsabilité. La condition de notre humanité, Paris, Autrement (série Morale 14).

Walzer M. (1997) Sphères de justice, Paris, Éditions du Seuil.

Wilson J.-Q., Kelling G.-L. (1994) Vitres cassées, Les cahiers de la sécurité intérieure, 15.

\section{NOTES}

1. PENNAC D., (Comme un roman) cité par L. DUBOUCHET « Une impossible définition ", Les jeunes en difficulté, dirigé par P DURET et A. GUY, Panoramiques $n^{\circ} 26$, 1996.

2. MAYOL P., « Les sept piliers de la culture jeune », Le Monde de l'Éducation, Juillet-Août, 1997.

3. « La cote sur le marché de la drague »... La valeur d'une liaison dépend de la valeur des partenaires antérieurs. JUHEM P, « Les relations amoureuses des lycéens », Sociétés contemporaines, « Les mondes des jeunes », n²1, 1995.

4. Le vieillissement social c'est « le lent travail de deuil, de désinvestissement qui porte les agents à ajuster leurs aspirations à leur chances objectives ... les conduisant à devenir ce qu'ils sont $»$.

5. Échapper à la mort est la preuve qu'une garantie règne sur son existence.

6. Chantal Nicole-Drancourt constate la disparition historique des « sphères intermédiaires » qui permettaient à la jeunesse de s'installer progressivement dans le salariat. Laurence Roulleau-Berger observe que dans des « espaces intermédiaires » de la ville des micro-lieux permettent aux jeunes des interactions culturelles et professionnelles.

7. Cf. la revue Migrant-Formation, L'École dans la ville: Ouverture ou Clôture, n97, juin 1994

8. A la suite de l'appel d'offre lancé par les ministères de l'éducation (DEP) et de l'intérieur (IHESI), coordonné par B. Charlot.

9. Enquête de « victimisation » effectuée dans le Doubs : manque de respect $48 \%$, affaires abîmées $28 \%$, chantage $16 \%$, coups $16 \%$, racisme $10 \%$, racket $4 \%$, agressions et harcèlement sexuel 3\%. Chiffres fournis lors de l'Université d'Été 1996: Violence à l'école, à la suite de l'appel d'offre lancé par les ministères de l'éducation (DEP) et de l'intérieur (IHESI) Paris VIII - Saint-Denis 8 au 12 juillet 1996, Doc Mafpen.

10. RICOEUR P., Temps et récit, T III, Le seuil, 1985, p. 339. Cité par J. Roman, «Entre Hannah Arendt et Eric Weil, Ricoeur, Esprit, 1988.

11. Pour Aristote l'équitable, tout en étant le juste, n'est pas le juste selon la loi, mais un correctif de la justice légale.

12. Pour Walzer il ne saurait y avoir un principe unique de justice, mais différents principes applicables dans des sphères spécifiques de la vie sociale.

13. Soit d'une part des personnes et de l'autre des formes de biens communs qui permettent d'établir une équivalence entre les êtres et de définir leur grandeur relative. Pour converger vers l'accord, les personnes font référence à quelque chose qui les dépasse, un principe d'équivalence qui leur permet de se justifier. 


\section{RÉSUMÉS}

Cette contribution à travers une approche de la violence scolaire interroge le mécanisme du lien scolaire dans sa complexité. Les actes de violence s'inscrivent dans un contexte, ils sont produits dans un cadre institutionnel et local, et ont un sens pour leurs auteurs et ceux qui les subissent. Ils définissent un état du lien scolaire. Mais la prise en compte du risque glisse parfois de la solidarité vers la sécurité de la responsabilité vers la fatalité. Au colloque de Villepinte 1997 (intitulé : «des villes sûres pour des citoyens libres »), l'inspecteur général J.-L. Nembrini déclarait: «on ne cherche pas l'ordre dans le quartier mais l'intégration des citoyens dans la nation, par consentement éducatif ». En dehors du tapage médiatique, il s'agit de resituer ces actes de violence et de participer à l'observation des relations entre les civilités et la citoyenneté scolaire ordinaire.

This contribution through scholar violence approach question scholar link mechanisms in its complexity. Violence acts take place in a context, they are produced in an institutional and specific frame and have sense for their actors and one who undergoes them. They define position of scholar link. But risk estimation slide sometimes from solidarity to safety, responsibility to fatality. At Villepinte symposium in 1997 (call "Safe towns for free citizens"), General Supervisor J.-L. Nembrini declared: “one doesn't want order in the area, but integration of citizens in Nation, by educative consent". Out of the noise of medias, it's necessary to situate once again violence and to participate to observation of relationship between civilized and ordinary scholar "citizenship".

\section{INDEX}

Mots-clés : école, jeunes, lien social

Keywords : school, violence, young people, social link

\section{AUTEUR}

\section{MARC BARTHÉLÉMY}

Professeur d'E.P.S. Collège St Jean de Védas. Equipe « Corps et Culture » 